\title{
INCORPORACIÓN DE ABONOS VERDES Y BIOFERTILIZANTE FOLIAR EN EL CULTIVO ORGÁNICO DE FRESA (Fragaria spp.) VARIEDAD BRITGET EN LAS SABANAS, MADRIZ
}

\author{
Álvaro Benavides González, J osé Cisne Contreras, Reinaldo Laguna Miranda \\ MSc. Docentes-Investigadores del Programa Recursos Genéticos Nicaragüenses, \\ Facultad de Agronomía, Universidad Nacional Agraria
}
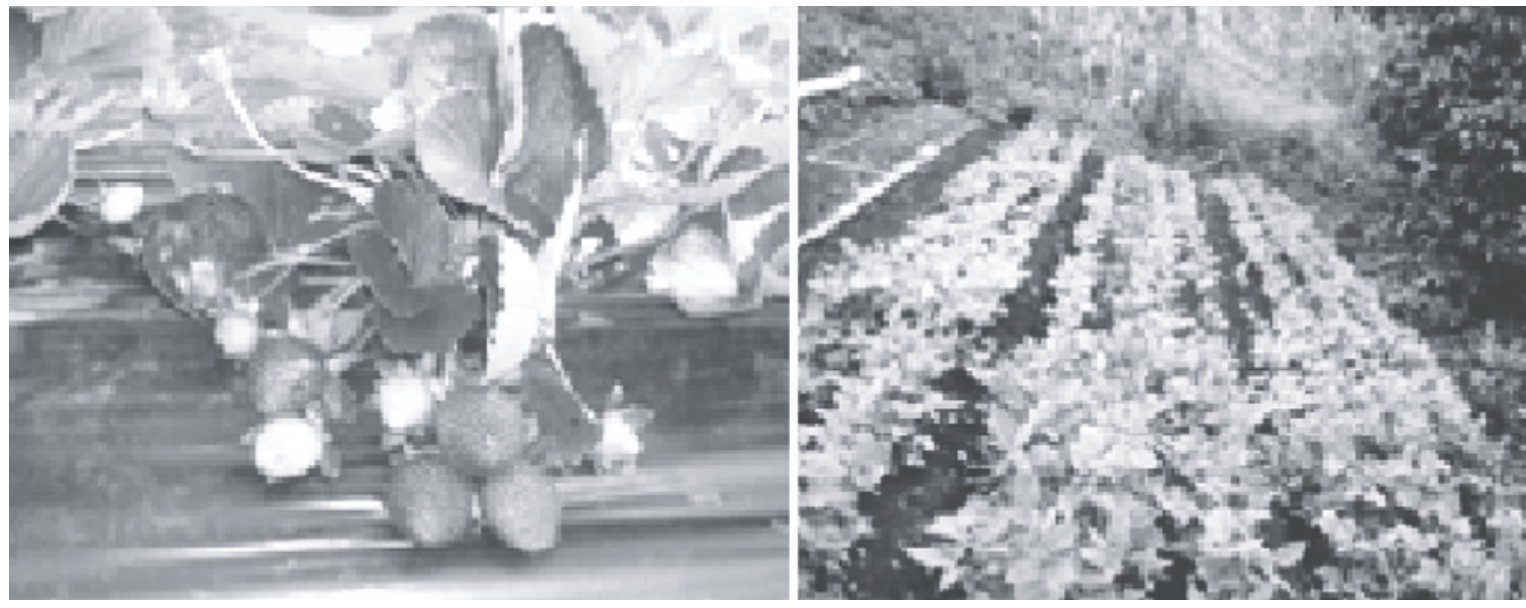

\section{RESUMEN}

Los pequeños productores de fresa del Municipio Las Sabanas, han venido desarrollando en conjunto con la Universidad Nacional Agraria, algunos trabajos que les han permitido incrementar la producción orgánica del cultivo de fresa (Fragaria spp.); sin embargo, las principales limitantes se centran sobre el manejo de plagas y enfermedades, fertilidad de los suelos, evaluación de nuevos genotipos, entre otros. Los objetivos del presente estudio fueron evaluar momentos de incorporación de abonos verdes y biofertilizante en el cultivar de fresa Britget. El estudio fue conducido en la finca del Sr. José Méndez, en la Comunidad El Castillito, Municipio de Las Sabanas, Departamento de Madriz durante el período de agosto del 2006 a mayo del 2007. Para esto se empleó un diseño de parcelas e franjas en el que se estudiaron especies de leguminosas: gandul (Vigna unguilata L.) y mungo (Vigna radiata L.), y su incorporación en el suelo (12, 24 y 36 dds), y niveles de biofertilizante foliar (200 y 400 $\left.1 \mathrm{ha}^{-1}\right)$. Los tratamientos resultaron del arreglo factorial 2x3x2 con tres réplicas. Las variables de fruto y rendimiento fueron sujeto a un análisis de varianza (ANDEVA) y categorización estadística mediante LSD $(\infty=0.05)$. Las variables de fruto fueron afectadas por las leguminosas incorporada al suelo. De igual manera, los resultados obtenidos indicaron que los tratamientos conformados tuvieron efecto significativo sobre el rendimiento total, no así en las interacciones. Los mayores
ABSTRACT
Strawberry (Fragaria spp.) small-scale cultivators from Las Sabanas, Madriz and the Universidad Nacional Agraria have developed several studies aimed to increase the organic production. Plagues and diseases management, soil fertility, new test of cultivars, among others, are the main limiting factors of the crop. The present study was carried out to evaluate the moment of green manure and biofertilizer incorporation in strawberry cv Britget. The study was conducted at the farm of Mr. Jose Méndez, El Castillito, Las Sabanas, during the period August 2006 - May 2007. The experiment was set up in a split-block design. Legume species cowpea (Vigna unguilata L.) and mungbean (Vigna radiata L.), their incorporation into the soil at 12, 24 and 36 days after planting (DAP), and foliar biofertilizer levels (200 and $400 \mathrm{l}$ $\mathrm{ha}^{-1}$ ) were evaluated. Treatments were arranged in a factorial experiment $(2 \times 3 \times 2)$ with three replicates. Fruit and yield variables were submitted to analysis of variance (ANOVA) and statistical categorization by LSD $(\infty=0.05)$. The legumes incorporated into the soil affected the fruit variables. The treatments, but not the interactions, had significant effect on total yield. In the principal effects, cowpea $\left(1490 \mathrm{~kg} \mathrm{ha}^{-1}\right)$ incorporated into the soil at 12 DAP $\left(1410 \mathrm{~kg} \mathrm{ha}^{-1}\right)$ and 400 $1 \mathrm{ha}^{-1}$ of biofertilizer (1318 $\mathrm{kg} \mathrm{ha}^{-1}$ ) resulted in the highest averages. Cowpea incorporated into the soil at 12 and 24 DAP with $400 \mathrm{l} \mathrm{ha}^{-1}$ of biofertilizer (2000 and $2100 \mathrm{~kg} \mathrm{ha}^{-1}$ of total 
valores promedios en los efectos principales se presentaron en caupí (1490 kg ha-1) a los 12 dds de incorporados al suelo

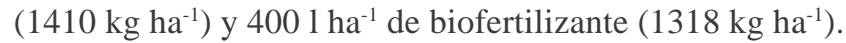
Los mejores tratamientos fueron el caupí incorporado al suelo a los 12 y 24 dds con 400 lts ha ${ }^{-1}$ de biofertilizante, con totales de 2000 y $2100 \mathrm{~kg} \mathrm{ha}^{-1}$ de fruta fresca, respectivamente.

Palabras clave: Fragaria, diseño parcelas subdivididas, Vigna unguilata, Vigna radiata, ANDEVA, LSD. fresh fruit respectively) resulted the best treatments.

Keywords: Fragaria, split- block design, Vigna unguilata, Vigna radiata, ANOVA, LSD.

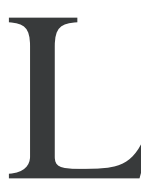

a fresa o frutilla (Fragaria spp.) es una planta que puede vivir varios años, sin embargo dura dos años en producción económica. El desarrollo científico y tecnológico de esta fruta ha contribuido a manejarla en condiciones de ambiente controlado. Sus características de forma, color, gusto y aroma, han hecho de la fresa uno de los productos más apetecidos, tanto para consumo directo como para la elaboración (Juscafresca, 1987). La diseminación de este cultivo por casi todo el mundo se debe al desarrollo de variedades con distinto grado de adaptación ecológica y a los modernos sistemas de manejo de cultivo, lo cual hace posible su producción desde las regiones frías hasta las regiones tropicales y subtropicales. La importancia actual que se ha dado en el mundo a la fresa ha hecho que su cultivo se extienda a casi toda Europa, principalmente en el Reino Unido, Francia, Alemania, ex-Yugoslavia, Países Bajos, Polonia y España. En América: Estados Unidos, Canadá, México, Guatemala, Costa Rica, Colombia, Ecuador, Chile y Argentina. Hay opiniones que sostienen que la fresa es uno de los productos con creciente posibilidad de expansión de consumo, incluso a mercados alejados que pueden ser abastecidos gracias al transporte aéreo (Bedoya, 2000); citado por Gómez (2006).

En 1993 se inició su cultivo nuevamente en el mismo departamento, y actualmente se siembra en otros departamentos del norte del país, incluyendo Matagalpa y Madriz (APENN, 1996). En las Sabanas Somoto, los productores tradicionalmente han cultivado café y hortalizas, y partir del año 2000 han experimentado con el cultivo de fresa como alternativa viable para mejorar su situación económica sin afectar el medio ambiente con rendimientos que superan los $8000 \mathrm{~kg} \mathrm{ha}^{-1}$ (Benavides et al., 2007). Se calcula que la demanda de algunas empresas en Nicaragua oscilan entre las 200 y 300 libras mensuales (IICA, 2007). El consumo nacional no está reportado, pero la agroindustria y supermercados tienen un consumo de 8 toneladas por semestre (Cruz, 2007). De acuerdo a información suministrada por técnicos y productores de Las Sabanas, los problemas que limitan la producción de fresa son: la identificación de variedades que se adapten a las condiciones edafoclimáticas de las zonas productoras, reproducción de material de siembra, manejo de enfermedades e insectos, así como problemas de comercialización del producto.

La Universidad Nacional Agraria y los productores de fresa de El Castillito, Las Sabanas, Departamento de Madriz han realizado investigaciones encaminadas a dar repuesta a algunos de los problemas que actualmente limitan la producción de fresa en dicha zona, por lo que el presente estudio pretende contribuir a mejorar la producción de fresa en sistemas de fincas orgánicas de pequeños agricultores evaluando especies de abonos verdes y biofertilizantes foliares.

\section{MATERIALES Y MÉTODOS}

Localización del área experimental. El experimento fue conducido en la Comunidad El Castillito, Municipio de Las Sabanas, Departamento de Madriz. Este departamento se encuentra ubicado en la región norte del país entre los $13^{\circ} 26^{\prime} 00^{\prime \prime}$ latitud norte y $86^{\circ} 37^{\prime} 01^{\prime \prime}$ longitud oeste. El Municipio de Las Sabanas limita al norte con el Municipio de San Lucas, al sur con San José de Cusmapa, al este con Estelí y al oeste con Honduras. Presenta una temperatura anual promedio entre 26 y 27 con una elevación de 1260 msnm. La Comunidad El Castillito presenta una temperatura anual entre 18 y 22. El clima es subtropical seco con precipitaciones anuales entre 1200 a $1400 \mathrm{~mm}$. El suelo presenta textura franco arenoso, $\mathrm{pH}$ neutro (6.74), materia orgánica alta (5.30\%), nitrógeno alto (0.26\%), fósforo pobre (7.21 ppm) y potasio alto (0.75 me/100g).

Tratamientos y diseño experimental. El material genético empleado fue la variedad Britget con procedencia de Guatemala, y mejorada en los Estados Unidos. El experimento se estableció sobre un diseño de Bloques Completos al Azar (BCA) con arreglo en Parcelas Subdivididas con tres replicaciones. En el estudio se evaluó el efecto de tres momentos de incorporación (12, 24 y 36 dds) de abono verde caupí (Vigna unguiculata L.) 
y mungo (Vigna radiata L.) y niveles de biofertilizantes (200 y $400 \mathrm{l} \mathrm{ha}^{-1}$ ). La siembra de la variedad es típica de los productores, estableciendo bancos lineales de 1x12 metros ancho y largo, respectivamente, con una altura de camellón de 0.30 metros. El método de siembra fue tres bolillos en el cual se apreciaron dos surcos La distancia entre surco y planta fue de 0.40 y 0.30 metros, respectivamente, y 0.80 metros entre cada banco o cantero. Las unidades experimentales fueron de $3 \mathrm{~m}^{2}$ para un total de $300 \mathrm{~m}^{2}$.

Establecimiento y manejo del experimento. El semillero fue establecido por los productores del 9 al 12 de agosto del 2006 contiguo a las parcelas experimentales. Se seleccionaron las plantas madres de mayor vigor y uniformidad, y se extrajeron del semillero las coronas. La preparación del terreno de siembra se efectuó del 15 de agosto al 31 de septiembre del 2006. El suelo se mulló, y se aplicó cal para la desinfección del suelo a razón de $2 \mathrm{~kg}$ por parcela, y se conformaron los camellones para la siembra. Posteriormente, fue tendido el plástico de polietileno negro calibre 1000 del 31 de agosto hasta el 11 de septiembre del 2006, esto debido a los momentos de incorporación del abono verde. Estando los bancales protegidos con el plástico negro, se procedió a realizar los agujeros sobre el plástico. Para conformar el sistema de siembra tres bolillos, se utilizó un triángulo de madera que puesto sobre el plástico y marcando los tres puntos se hace el agujero con una navaja, utilizando la el diámetro que delimita la una botella de plástico de 1.5 litros cortada de manera transversal. El mungo ( $V$. radiata L.) y caupí (V. unguiculata L.) fue sembrado a partir del 14 de agosto del 2006. Posteriormente, se mulló y se incorporo al suelo de manera mecánica con el azadón, y las leguminosas se fueron incorporando al suelo a los 12, 24 y 36 después de la siembra (dds). Provenientes del semillero, las plántulas fueron desinfectadas con cloro al $5 \%$, y establecidas en cada agujero 6 días después de incorporar el abono verde.

La poda sanitaria y de forma fue realizada durante los meses de agosto y diciembre del 2006. Las hojas secas y enfermas fueron eliminadas de las parcelas experimentales. Asimismo, el control de malezas se realizó en el mes de diciembre del 2006, y las malezas dentro de los orificios fueron extraídas de forma manual, y las que crecieron fuera de este, fueron controladas con machete y azadón.

La fertilización orgánica empleada fue consensuada con el productor y tomando en cuenta las características de los suelos. Se aplicó lombrihumus a razón de 2 libras por $\mathrm{m}^{2}$ (3000 $\left.\mathrm{kg} \mathrm{ha}^{-1}\right)$ al inicio de la siembra y de 1 a $2 \mathrm{l}$ de biofertilizante foliar (200 y $400 \mathrm{~kg} \mathrm{ha}^{-1}$ ) durante los meses después de la siembra hasta enero del 2007 a intervalos de 15 días. Por otro lado, el riego se aplicó entre enero a abril del 2007 y se regó dos veces por semana.

El biofertilizante foliar fue preparada a partir de 9.1 kg de estiércol fresco, 40 l de suero y $40 \mathrm{l}$ de melaza en 601 de agua dejándose fermentar por 22 días para su posterior uso.

La eliminación de flores y estolones se realizó simultáneamente durante los meses de octubre y noviembre del 2,006, cada siete días en estado de botón o recién abierta las flores, y se finalizó cuando la planta había formado de 4 a 5 coronas.

Variables evaluadas y análisis de datos. La longitud y ancho del foliolo central de la hoja trifoliada se midieron con regla en centímetro en la parte longitudinal y central, respectivamente, en cinco plantas de la unidad experimental.

La longitud, diámetro y espesor del fruto se midieron con vernier en la parte longitudinal, frontal y lateral del fruto, respectivamente. Para la cosecha se utilizó balanza, baldes y cajas parar el empaque. Los frutos frescos de cada planta y las parcelas cosechadas de enero a mayo del 2007, fueron pesados y calculados su rendimiento a kg ha-1. El productor realizó otras cosechas en menor escala que no fueron incluidas.

La información fue manejada en hojas electrónicas (Excel) para su posterior análisis con SAS (v. 9.1). Se realizó análisis de varianza (ANDEVA) sobre las variables, y categorización estadística (LSD, $\infty=0.05$ ).

\section{RESULTADOS Y DISCUSIÓN}

Algunas de las características de crecimiento, desarrollo y de rendimiento en el cultivo de fresa pueden ser afectadas por las técnicas de manejo agronómico, el material genético y los tratamientos evaluados (Guerena et al., 2003; Toledo, 2003). Benavides et al., 2007), encontraron que niveles de lombrihumus aplicados al suelo, así como diferentes dosis de biofertilizantes asperjados a las hojas cada 15 días antes y durante las cosechas, presentaron efecto significativo en las cosechas obtenidas. De igual manera, Cruz (2007), encontró diferencias estadísticas en variables de crecimiento, de frutos y de rendimiento al utilizar 3 distancias de siembra sobre el cultivo de fresa.

El rendimiento total de fruta fresca fue afectado significativamente por las leguminosas y sus momentos de incorporación al suelo, así como los niveles de biofertilizante foliar. No se encontró significación estadística en las interacciones conformadas (Tabla 1). Las mayores varianzas de los errores se obtuvieron en 
la incorporación de leguminosas ( $\mathrm{DE}=491.91)$, seguido de las aplicaciones de biofertilizante foliar (DE=291.04) y leguminosas ( $\mathrm{DE}=166.36)$. Asimismo, la aplicación de biofertilizante tuvo efecto significativo en los meses de febrero, marzo y mayo; resultados similares se presentaron en los abonos verdes y su incorporación al suelo en el mes de enero (Tabla 1). biofertilizante fue efectiva estadísticamente, siendo inferior en un $27 \%$ el rendimiento total cuando se asperjó $200 \mathrm{l} \mathrm{ha}^{-1}$. Benavides et al., (2007), reportan incrementos significativos del $20 \%$ en rendimiento total de fruta fresca cuando hicieron uso de $400 \mathrm{l} \mathrm{ha}^{-1}$ sobre $200 \mathrm{l} \mathrm{ha}^{-1}$ (Tabla 2). Las leguminosas incorporadas al suelo tuvieron efecto significativo en las cosechas obtenidas en los meses de enero, abril y mayo, superando

Tabla 1. Desviación estándar del error (DE) y significación estadística del rendimiento mensual y total de los factores evaluados en el cultivar de fresa Britget durante 2007. El Castillito, Las Sabanas, Madriz

\begin{tabular}{|c|c|c|c|c|c|c|c|c|}
\hline Variantes & enero & & febrero & marzo & abril & mayo & Total & \\
\hline Bloque & 793.8 & $*$ & 173.5 & 72.7 & 92.9 & 76.5 & 358.2 & \\
\hline Leguminosa (Leg) & 395.9 & $*$ & 512.7 & 199.5 & $295.1 *$ & $206.4 *$ & 1967.0 & $*$ \\
\hline Incorporación (Inc) & 598.0 & $* *$ & 275.5 & 217.2 & 100.4 & 109.9 & 1487.8 & $* *$ \\
\hline Leg*Inc & 226.2 & & 292.6 & 124.0 & 17.2 & 45.2 & 395.0 & \\
\hline Biofertilizante (Bio) & 245.9 & & $289.7 *$ & $243.7 *$ & $95.6 *$ & $139.4 *$ & 1062.7 & $* *$ \\
\hline Leg*Bio & 19.0 & & 195.3 & 48.1 & 72.5 & 88.9 & 503.0 & \\
\hline Inc*Bio & 143.9 & & 182.0 & 134.0 & 76.0 & 102.7 & 226.2 & \\
\hline Leg*Inc*Bio & 377.2 & & 148.8 & 56.2 & 86.7 & 37.4 & 175.7 & \\
\hline $\mathrm{R}^{2}$ & 0.862 & & 0.904 & 0.832 & 0.829 & 0.871 & 0.930 & \\
\hline CV (\%) & 29.3 & & 26.3 & 16.4 & 28.6 & 25.8 & 24.9 & \\
\hline
\end{tabular}

$*, * *=$ Significativo a los niveles de $\infty=0.05 \mathrm{y} \infty=0.01$, respectivamente

Rendimiento mensual y total. Davis (1985), afirma que el rendimiento en los cultivos es un carácter continuo influenciado por factores edáficos y ambientales. De igual manera, está determinado por el genotipo, la ecología y el manejo agronómico de la plantación (Blandón y Arvizú, 1991; citado por Sequeira, 2004).

El rendimiento total de fruta fresca varió significativamente en las leguminosas, superando el caupí al mungo en un 34 \%. En los momentos de incorporación de las leguminosas, se logró diferenciar a través de LSD $(\infty=0.05) 2$ grupos: el grupo conformado por los momentos 12 y 24 dds, y el de 36 dds; en donde la incorporación a los 24 dds superó en 1.72 \% a la incorporación 12 dds. La incorporación realizada a los 12 y 24 dds superó significativamente en 43 \% y $42 \%$, respectivamente, al momento 36 dds. La aplicación de el caupí al mungo en más del 25 \%. La incorporación de las leguminosas al suelo fue efectiva solamente en los meses de enero y abril. De igual manera, la aplicación de biofertilizante presentó significación estadística en los meses de febrero, marzo, y mayo, con promedios que superaron la aplicación de $200 \mathrm{l} \mathrm{ha}^{-1}$ en 15 \%, 31\% y 41 $\%$, respectivamente (Tabla 2).

En Nicaragua, APENN (1996) reporta rendimientos de $3882 \mathrm{~kg} \mathrm{ha}^{-1}$. En los estudios experimentales realizados en El Castillito, Las Sabanas, se han obtenido rendimientos de $848 \mathrm{~kg} \mathrm{ha}^{-1}$ (Espinoza, 2007), $7445 \mathrm{~kg}$ ha $^{-1}$ (Cruz, 2007), y $8200 \mathrm{~kg} \mathrm{ha}^{-1}$. Estos reportes en su mayoría, son superiores a los obtenidos en la Tabla 2, debido principalmente a que el presente experimento se estableció dos meses después de lo apropiado. 
Tabla 2. Categorización estadística del rendimiento en $\mathrm{kg} \mathrm{ha}^{-1}$ mensual y total en los niveles de los factores evaluados en el cultivar de fresa Britget durante 2007. El Castillito, Las Sabanas, Madriz.

\begin{tabular}{|c|c|c|c|c|c|c|c|}
\hline Variantes & Nivel & enero & febrero & marzo & abril & mayo & Total \\
\hline \multirow[t]{2}{*}{ Leguminosa } & Caupí & $414.1 \mathrm{a}$ & 889.7 a & $459.1 \mathrm{a}$ & 765.2 a & 207.4 a & $2735.5 \mathrm{a}$ \\
\hline & Mungo & 207.3 b & 595.7 a & 341.4 a & 568.9 b & $91.4 \mathrm{~b}$ & $1804.7 \mathrm{~b}$ \\
\hline \multirow[t]{3}{*}{ Incorporación } & 12 dds & $299.5 \mathrm{ab}$ & $852.5 \mathrm{a}$ & $466.5 \mathrm{a}$ & $777.5 \mathrm{a}$ & 186.4 a & $2582.3 \mathrm{a}$ \\
\hline & $24 \mathrm{dds}$ & 497.6 a & $742.5 \mathrm{a}$ & $427.8 \mathrm{a}$ & $713.1 \mathrm{a}$ & 156.9 a & $2537.8 \mathrm{a}$ \\
\hline & 36 dds & $125.3 \mathrm{~b}$ & $611.1 \mathrm{a}$ & $232.2 \mathrm{a}$ & $386.9 \mathrm{~b}$ & $110.8 \mathrm{a}$ & $1466.3 \mathrm{~b}$ \\
\hline \multirow[t]{2}{*}{ Biofertilizante } & $200 \mathrm{l} \mathrm{ha}^{-1}$ & 276.3 a & $678.0 \mathrm{~b}$ & $330.0 \mathrm{~b}$ & $550.0 \mathrm{~b}$ & $115.4 \mathrm{~b}$ & $1949.6 \mathrm{~b}$ \\
\hline & $400 \mathrm{l} \mathrm{ha}^{-1}$ & 389.5 a & $800.2 \mathrm{a}$ & $478.3 \mathrm{a}$ & $797.2 \mathrm{a}$ & 197.5 a & 2662.6 a \\
\hline
\end{tabular}

Medias con letras semejantes no difieren estadísticamente $(\mathrm{LD}, \infty=0.05)$

En cuanto a variables del foliolo central, no se observó efecto significativo $(\operatorname{Pr}>0.05)$ en las leguminosas y biofertilizante, no así en los momentos de incorporación (Tabla 2). Los mayores valores de diámetro y longitud de foliolo se presentaron en la incorporación de biofertilizante cuando se incorporó a los 12 dds y 36 dds, resultados estadísticos similares se obtuvieron en las dimensiones del fruto (Tabla 2). Téllez y Salmerón (2007), y Cruz (2007), reportan valores promedios de longitud del foliolo de $4.56 \mathrm{~cm}$ y de $6.58 \mathrm{~cm}$, y longitud promedio de fruto de $37.53 \mathrm{~mm}$ para la variedad Britget, superando a los valores obtenidos en la Tabla 3.
La incorporación de las leguminosas mungo y caupí en la siembra del cultivo de fresa, mostraron evidencias significativas en la variación de los descriptores del foliolo y longitud del fruto. El rendimiento total obtenido en las 5 cosechas también fue afectado por los momentos de incorporación del caupí y mungo (Tabla 2). Estos resultados se le atribuyen a la rápida descomposición y liberación de nitrógeno de las especies en estudio. García (2006), reportó el máximo porcentaje de descomposición en el suelo después de incorporado a las 5 y 7 semanas para las leguminosas caupí y mungo, respectivamente.

Tabla 3. Categorización estadística del rendimiento en $\mathrm{kg} \mathrm{ha}^{-1}$ mensual y total en los niveles de los factores evaluados en el cultivar de fresa Britget durante 2007. El Castillito, Las Sabanas, Madriz

\begin{tabular}{|c|c|c|c|c|c|c|}
\hline \multirow[b]{2}{*}{ Factor } & \multirow[b]{2}{*}{ Nivel } & \multicolumn{2}{|c|}{ Folíolo central $(\mathrm{cm})$} & \multicolumn{2}{|c|}{ Fruto (mm) } & \multirow[b]{2}{*}{ Longitud } \\
\hline & & Diâmetro & Longitud & Diámetro & Espesor & \\
\hline \multirow{2}{*}{ Leguminosa } & Caupí & $5.70 \mathrm{a}$ & $7.00 \mathrm{a}$ & 25.94 а & $23.40 \mathrm{a}$ & 31.77 a \\
\hline & Mungo & 5.60 a & 6.76 а & $25.21 \mathrm{a}$ & $22.81 \mathrm{a}$ & $31.71 \mathrm{a}$ \\
\hline \multirow[t]{3}{*}{ Incorporación } & $12 \mathrm{dds}$ & $5.69 \mathrm{ab}$ & $7.03 \mathrm{a}$ & 23.70 a & $22.10 \mathrm{a}$ & $29.06 \mathrm{~b}$ \\
\hline & $24 \mathrm{dds}$ & $5.24 \mathrm{~b}$ & $6.41 \mathrm{~b}$ & 26.87 a & $23.81 \mathrm{a}$ & 33.30 a \\
\hline & $36 \mathrm{dds}$ & 5.98 а & $7.20 \mathrm{a}$ & 26.12 a & 23.36 a & $32.80 \mathrm{a}$ \\
\hline \multirow[t]{2}{*}{ Biofertilizante } & $200 \mathrm{l} \mathrm{ha}^{-1}$ & $5.70 \mathrm{a}$ & 6.80 a & 26.14 a & $23.84 \mathrm{a}$ & 32.92 a \\
\hline & $400 \mathrm{l} \mathrm{ha}^{-1}$ & $5.60 \mathrm{a}$ & 6.94 a & 25.02 a & $22.36 \mathrm{a}$ & 30.55 a \\
\hline
\end{tabular}

Medias con letras semejantes no difieren estadísticamente (LSD, $\infty=0.05)$ 


\section{CONCLUSIONES}

Basado en los resultados del presente estudio en el cultivo de fresa, se deduce lo siguiente.

Las leguminosas caupí y frijol mungo y sus momentos de incorporación al suelo, así como los niveles de biofertilizantes en la variedad Britget mostraron efecto significativo sobre variables de crecimiento y rendimiento. Asimismo; no se encontró interacción alguna en los efectos principales.

El caupí incorporado a los 12 y 24 dds, y la aplicación de 4001 ha $^{-1}$ de biofertilizante determinaron los mayores rendimientos totales con promedios superior a los 2000 $\mathrm{kg} \mathrm{ha}^{-1}$ en cinco cosechas realizadas, obteniéndose los mayores promedios en los meses de febrero y abril.

\section{AGRADECIMIENTO}

Se agradece la colaboración especial del señor José Méndez y a la Universidad Nacional Agraria, que a través de fondos proveídos por el pueblo y gobierno de Suecia a través de Asdi, y administrado por la DIEP permitió el desarrollo de la presente investigación.

\section{REFERENCIAS BIBLIOGRÁFICAS}

APENN (Asociación Nicaragüense de Productores y Exportadores de Productos no Tradicionales). 1996. Nicaragua for Export. Revista del exportador. Managua, Nicaragua. p. 22-23.

Benavides, G; Cisne, J; Laguna, R. 2007. Fertilización orgánica sobre tres genotipos de fresa (Fragaria spp.) en Las Sabanas. La Calera, Año 7, Nº. 8, Mayo 2007. Universidad Nacional Agraria. Managua, Nicaragua. p. 54-58.

Cruz, GT. 2007. Efecto de tres distancias de siembra y tres alternativas de manejo de enfermedades en el cultivo orgánico de fresa (Fragaria ssp.) variedad Festival, en Las Sabanas, Madriz. Tesis de Ing. Agr., Universidad Nacional Agraria (UNA) Managua, Nicaragua. p. 7

Davis, CH; 1985. Conceptos básicos de genética de frijol. En frijol: Investigación y producción. CIAT. Calí, Colombia, p 81-87.

Espinoza, B. 2007. Diagnóstico y dinámica de enfermedades en tres genotipos de fresa (Fragaria spp.) en la comunidad El Castillito, las sabanas departamento de Madriz. Tesis de Ing. Agr., Universidad Nacional Agraria (UNA) Managua, Nicaragua. p. 1-2.

García, CL. 2006. Uso de abonos verdes en cultivos agrícolas. Guía técnica No. 10. Universidad nacional Agraria (UNA) Managua, Nicaragua. p. 5-13.

Gómez, JA. M. 2006. Descripción del comportamiento de insectos y enfermedades asociadas al cultivo de fresa (Fragaria spp.) en el municipio de La Sabana, departamento de Madriz. Tesis de Ing. Agr. Universidad Nacional Agraria. Managua, Nicaragua. 101 p.

Guerena M; Ames, G; Born, H. 2003. Fresas orgánicas y opciones para el manejo integrado de plagas: Guía de producción hortícola. Centro Nacional de Tecnología Apropiada (NCAT). Appropiate Technology Transfer for Rural Areas (ATTRA). 23 p.

IICA (Instituto Interamericano de Cooperación para la Agricultura). 2007. FRESA: Guía práctica para la exportación a EE.UU. 12 p.

Juscafresca, B; Ibar, L. 1987. Fresas y fresones. Editorial Aedos, biblioteca agrícola AEDOS. 39 p.

Sequeira R. GA. 2004. Evaluación de diferentes porcentajes de lombrihumus y suelo, como sustrato en la producción de posturas de chiltoma (Capsicum annum L.) en bandejas para trasplante. Tesis de Ing. Agr. Universidad nacional Agraria. Managua, Nicaragua. 41 p.

Tellez, F; Salmerón, L. 2007. Efecto de cuatro niveles de fertilización orgánica sobre tres variedades de fresa (Fragaria sp.) En las Sabanas, Madriz. Tesis de Ing. Agr., Universidad Nacional Agraria (UNA) Managua, Nicaragua. p. 1-2.

Toledo, M. 2003. Guía para la Producción de Fresa en Honduras. Fundación Hondureña de Investigación Agrícola (FHIA). Junio, 2003. La Esperanza, Intibucá, Honduras, C. A. 36 p. 